\title{
Debugging Large-Scale, Long-Running Parallel Programs
}

\author{
Dieter Kranzlmüller, Nam Thoai, and Jens Volkert \\ GUP Linz, Johannes Kepler University Linz, \\ Altenbergerstr. 69, A-4040 Linz, Austria/Europe, \\ kranzlmueller@gup.uni-linz.ac.at, \\ http://www.gup.uni-linz.ac.at/
}

\begin{abstract}
Cyclic debugging depicts error detection techniques, where programs are iteratively executed to identify the original reason for incorrect runtime behavior. This characteristic is especially problematic for large-scale, long-running parallel programs concerning the requirements in time and processing resources and the associated computing costs. A solution to these problems is offered by a combination of techniques, which use the event graph model as the main representation of parallel program behavior. On the one hand, the number of deployed processes can be reduced with process isolation, where only a subset of the original processes are executed during debugging. On the other hand, an integrated checkpointing mechanism allows to extract limited periods of execution time, or to start subsequent program executions at intermediate points. Additionally, the event graph offers equivalent program execution in case of nondeterminism, as well as the possibility to investigate the effects of program perturbation induced by the observation functionality.
\end{abstract}

\section{Introduction}

Debugging is widely accepted as an important part of the software lifecycle, which tries to improve a program's reliability (and thus quality) by location and correction of errors. As soon as failures or incorrect results are observed, activities to locate the original source of the anomaly and to eliminate the reason for the incorrect behavior are initiated. Unfortunately, the existence of a bug in a program does not imply knowledge about the error(s) behind it [14]. In fact, the goal of debugging is to deduce the conditions under which the program produced the incorrect output [1]. This characterizes debugging as a "backward" process, and the corresponding activity is called flowback analysis [2]. Due to the impracticality of reverse execution - some statements, such as manipulation of file pointers and I/O descriptors, are usually irreversible [7] - flowback analysis is applied through cyclic debugging.

During cyclic debugging, repeated program executions are utilized to increase the user's knowledge about the application's behavior. This is achieved by placing breakpoints somewhere in the code, which halt a program at runtime and allow inspection of the obtained state [4]. Since it is a priori unknown, whether a breakpoint is really adequate and useful, the placement of breakpoints includes a certain amount of guesswork. In case a breakpoint is required prior to the reached state - somewhere in the past 
of a program's execution - another program run must be initiated from the very beginning. Often, several executions are required to identify the very reason for erroneous behavior.

In order to support these program analysis activities, many useful debugging tools for sequential and parallel software have been developed. Some well-known examples are the command-line debuggers $g d b$ and $d b x$, which are included in many operating systems. In addition, advanced debugging tools often incorporate instances of $g d b$ and $d b x$ for the core debugging activities. Example debuggers in this domain include $x x g d b$ or $D D D$ [24]. Some representative parallel debugging tools include $P 2 D 2$ [9], Panorama [16], PDBG [5], and the commercial debugger Totalview [6], which enrich the capabilities of sequential debuggers with special features for dealing with parallel code.

A particular challenging problem of parallel debuggers is given by the possible scale and the runtime-requirements of parallel software. Parallel and distributed computing is often proposed as the only means to attack computational intensive problems, because sequential computing does not offer sufficient performance. This is especially true for applications of computational science and engineering (CSE), which manifest their need for parallel computing through their requirements in computational speed, amount of memory, and accuracy of results [10]. As a consequence, numerous CSE applications utilize large numbers of processes over a substantial amount of time - days, weeks, or even month - to solve fundamental problems with the available computational resources.

The problem of debugging large-scale, long-running parallel programs is addressed by the approach described in this paper. The principal idea is comparable to program slicing, which reduces a given program by decomposing its execution according to the observed program and data flow [22]. The important idea is to concentrate only on the most important parts of a program - those containing the error - and ignore all other parts. This kind of abstraction is achieved with the event graph model, which represents a particular program execution [11]. Based on this graph, the actually required number of processes during debugging can be reduced to manageable amounts, while the "surroundings" of the selected processes are simulated by the system. For reductions of a program's runtime, the event graph is used as a basis for identifying suitable checkpoint lines. These checkpoints are established to allow the initiation of a program's execution at certain intermediate points instead of the very beginning of the original execution.

This paper is organized as follows: Section 2 defines the event graph and its generation for a parallel program execution with a given set of input data. Some details about reduction of participating processes are given in Section 3, while the combination of checkpointing and debugging is described in Section 4. Some optional extensions to the basic debugging strategy are discussed in 5, before a summary and an outlook on future work in this project concludes the paper.

\section{Generating the Event Graph of a Program's Execution}

The primary goal of our debugging strategy is reduction of the number of processes and the required execution time without affecting a program's original behavior. Therefore 
the traditional solution of down-scaling [8] by using smaller problem-sizes with less processes is not feasible, since the behavior of the program may be substantially different for varying problem sizes. In some cases, erroneous behavior may only occur on large scales, and testing with corresponding numbers of processes and problem sizes is therefore inevitable.

A prerequisite for our kind of reduction is exact knowledge of the relations between processes within a parallel program. The relations are captured by the abstract event graph model [11], which is a directed graph $G=(E, \rightarrow)$. The non-empty set of events $E$ is comprised of the events $e_{p}^{i}$ of $G$ observed during program execution, with $i$ denoting the sequential order on process $p$. An event itself is only a virtual item, because it does not require any time for its existence, but instead defines the instant when something happens [20]. It appears to mark a certain activity over the course of time, and therefore emphasizes the activities that are going on [3].

Anything or, to be precise, any state changes of interest occurring during program execution can trigger an event. In particular, the events of interest are established through communication and synchronization statements, which connect events on distinct processes. In parallel programs, process interaction is established either via shared memory or via message passing. The former uses accesses to a common address space to interchange information, while the latter applies dedicated message transfer functions. Although the abstract characteristics of the event graph model allow its application to both kinds of programming paradigms [11], this paper focuses only on the message passing paradigm, which is more scalable due to its loose coupling of processes. The corresponding set of events $E$ consists of communication and synchronization events, as e.g. generated at send and receive statements. In practice, the described approach has been implemented for point-to-point communication functions of the standard message passing interface MPI [17].

The relation " $\rightarrow$ " connecting two events $e_{p}^{i}$ and $e_{q}^{j}$ is the "happened-before" relation [15]. The expression $e_{p}^{i} \rightarrow e_{q}^{j}$ describes that there is an edge from event $e_{p}^{i}$ to event $e_{q}^{j}$ in $G$ with the "tail" at event $e_{p}^{i}$ and the "head" at event $e_{q}^{j}$. In concrete, $\rightarrow$ is the transitive, irreflexive closure of the union of the sequential order relation $\stackrel{S}{\rightarrow}$ and the concurrent order relation $\stackrel{C}{\rightarrow}$. The former is implicitly provided as index of each event relative to a particular process, where $e_{p}^{i} \stackrel{S}{\rightarrow} e_{p}^{i+1}$ means, that the $i^{\text {th }}$ event $e_{p}^{i}$ on any (sequential) process $p$ occurred before the $i+1^{\text {th }}$ event $e_{p}^{i+1}$ on the same process $p$.

The important relation is the concurrent order relation $\stackrel{C}{\rightarrow}$, which represents interprocess communication and coordination. The concurrent order of events $e_{p}^{i} \stackrel{C}{\rightarrow} e_{q}^{j}$ defines, that the $i^{t h}$ event $e_{p}^{i}$ on any process $p$ occurred directly before the $j^{t h}$ event $e_{q}^{j}$ on another process $q$, if $e_{p}^{i}$ is the sending of a message by process $p$ and $e_{q}^{j}$ is the receipt of the same message by another process $q$, or $e_{p}^{i}$ affects the behavior of $e_{q}^{j}$.

Within the event graph, $e_{p}^{i} \rightarrow e_{q}^{j}$ means, that $e_{p}^{i}$ preceded $e_{q}^{j}$ and $e_{q}^{j}$ occurred after $e_{p}^{i}$. An important characteristic for debugging is that $e_{p}^{i} \rightarrow e_{q}^{j}$ describes the possibility for event $e_{p}^{i}$ to causally affect event $e_{q}^{j}$, or in other words, event $e_{q}^{j}$ may be causally 
affected by event $e_{p}^{i}$. An event $e_{q}^{j}$ is causally affected by event $e_{p}^{i}$, if the state of process $q$ at event $e_{q}^{j}$ depends on the operation carried out at event $e_{p}^{i}$.

In order to use the event graph for debugging, a parallel program has to be instrumented by adding monitoring code to its executable. Each statement responsible for an event of interest is extended by probes, which deliver necessary information about the occurrence of the event and its relations. During execution of the program with a given input, the probes extract required event graph information and store it in tracefiles for post-mortem processing. This information consists of the following two data structures corresponding to events and relations:

$$
\begin{gathered}
\text { event: } e_{p}^{i}=(p, i, \text { type, data }) \\
\text { happened-before relation: } e_{p}^{i} \rightarrow e_{q}^{j}=(p, i, q, j)
\end{gathered}
$$

The variables $p$ and $i$ identify the process on which the event occurred and its relative sequential order, respectively. The type identifies the kind of observed event, which is either a send or receive operation. The data field can be used for optional attributes, which are empty in the event graph's most basic form. Please note, that determining the happened-before relation requires some kind of logical clock algorithm as described in [15].

After obtaining the event graph data, a debugging tool may use the data for arbitrary analysis activities. A common practice is the visualization of the event graph as a spacetime diagram, with the processes on one axis and the run-time on the second axis. An example space-time diagram for a program's execution is shown in Figure 1. The target program is a finite element solver, which has been executed on 64 processes. The processes are visualized vertically, while the time is displayed along the horizontal axis. Process interaction is visualized with directed edges connecting corresponding sender and receiver operations. The total number of events in this example is 40.000 over approx. 100 seconds of execution time.

As shown in Figure 1, the limits of the display permits only a reduced number of processes and a limited fraction of the program's execution to be visible at a time. Other areas of the event graph can only be investigated through standard graphical manipulations such as zooming and scrolling. In contrast, the techniques described in this paper try to reduce the actual extensions of the event graph in both dimensions. The process isolation approach described in Section 3 reduces the number of processes contained in the event graph. With an integrated checkpointing mechanism, the runtime of the parallel program can be limited as described in Section 4. While these techniques are primarily intended as a reduction of the requirements in terms of computational resources, they also help to simplify the visual representation.

\section{Process Isolation Technique}

The process isolation approach is initiated, when the reason for erroneous behavior is expected to be restricted to a small subset of all processes. As a starting point, only the actual process exhibiting the error may be a target for process isolation [12]. Whenever backtracking (by repeated executions) detects more and more process interactions, 


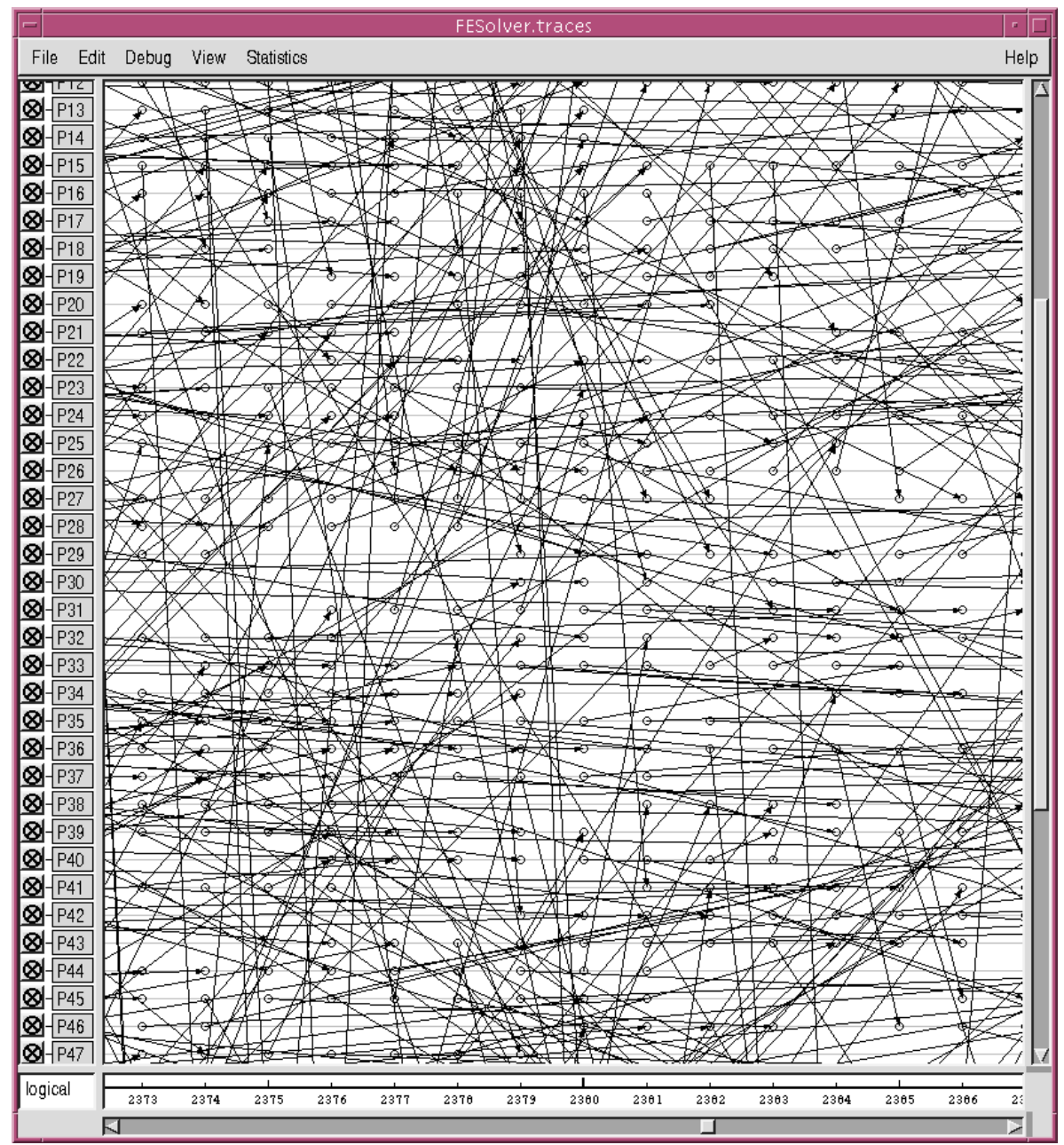

Fig. 1. Example event graph of a parallel program's execution

the number of isolated processes may be increased. In fact, the actual set of processes depends on the suspected distance between the observation point of the erroneous behavior and its original reason, as well as the number of relations to other processes. This characteristic defines the requirements of the process isolation strategy, namely to identify the set of processes and their relations within the given time interval.

The process isolation strategy is initiated by specifying which processes are to be extracted. Based on this specification, the event graph model of the program's execution is analyzed to identify the data required to simulate the surroundings of the selected process group. In case of message passing programs, the interesting data represents all messages, that have been transferred to the process group under consideration. This means that whenever a process of this target group performs a receive operation, the incoming data needs to be available for simulating the process without the original 


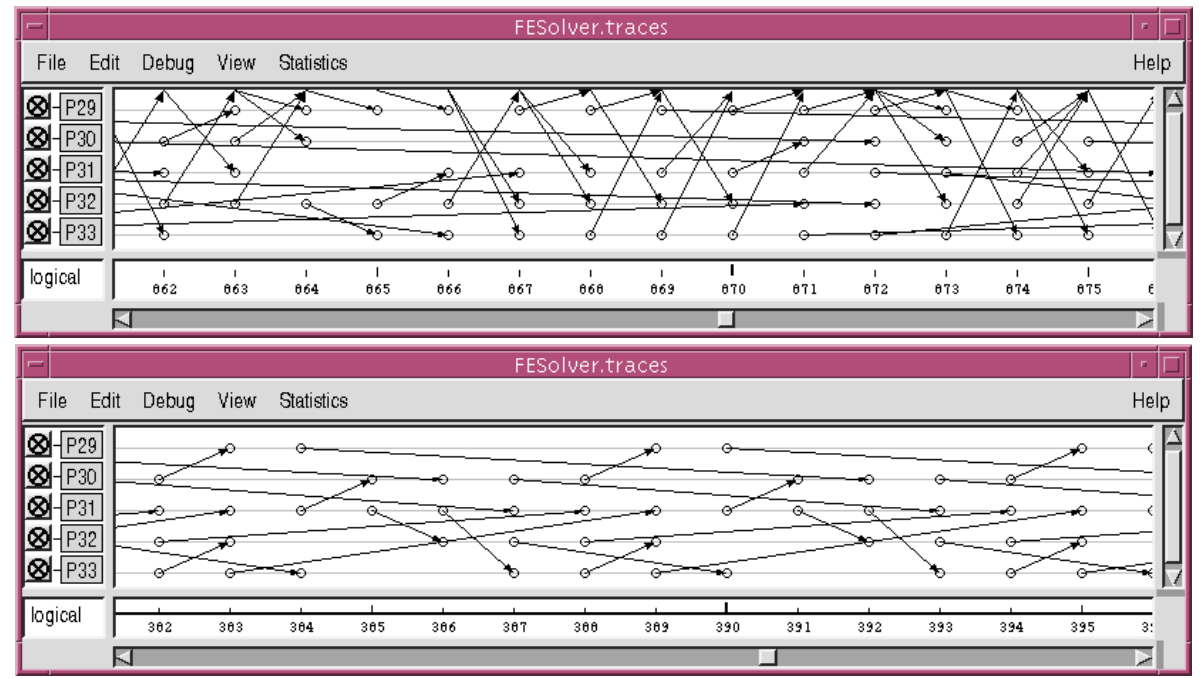

Fig. 2. Example process (group) isolation

sender. Please note, that all intragroup communication between the isolated processes is not required, because it will be generated on the fly.

In order to obtain the required data, the amount of instrumentation is increased at all receive statements. Identifying these receive statements based on the event graph model is rather trivial. During another execution of the program, the monitoring code checks whether the process belongs to the isolated group or not. In case the process belongs to the group, the origin of a incoming message is checked, and only if the origin of the message is outside the group, the actual message data is stored in tracefiles for subsequent analysis phases. In all other cases, the message data can be neglected:

$$
\text { event: } e_{p}^{i}=(p, i, \text { type, message })\left\{\begin{array}{l}
p \in \text { group } \\
\operatorname{type}\left(e_{p}^{i}\right)=\text { "receive" } \\
\operatorname{origin}\left(\operatorname{message}\left(e_{p}^{i}\right)\right) \notin \operatorname{group}
\end{array}\right.
$$

With the original event graph and the additional data of the process isolation phase, subsequent executions of only the limited numbers of processes are possible. The correct behavior of these processes will be transparently simulated by the underlying execution environment. This is achieved by intercepting all communication functions and controlling all operations affecting other processes. Process interaction is carried out as specified in the event graph. If data is transferred from outside the isolated process group to a member of the group, the message is obtained from the tracefile and handed to the target process. If data is transferred within the group, regular communication is carried out. All data originating in the group with a destination to a process outside the group can be discarded. Besides, the execution environment must take care of the original process numbering by using virtual process numbers during re-execution.

An example for the process isolation technique is given in Figure 2. The event graph under consideration is the same as in Figure 1, but the number of processes has been 
limited to a subset of 5 processes (instead of 64 as in the original execution). The top display shows all the communication of the 5 selected processes. The communication operations with messages from processes outside the isolated group are displayed with edges from/to the top of the space-time diagram. These messages are read from the traces instead of transmitting them, when the processes are isolated. The bottom diagram shows the same event graph and the same process group, but only those (intragroup) messages, that are actually transferred between the processes.

\section{Combining Checkpointing and Debugging}

In contrast to the process isolation approach, the integrated checkpointing approach attempts to reduce the actually required execution time. The term checkpointing describes the act of saving the state of a running program, so that it may be reconstructed from that position later in time [19]. In practice, checkpointing is usually applied to faulttolerant computing; whenever a program's execution fails, the execution of a program may proceed from the last available checkpoint.

The application of checkpointing for debugging large-scale parallel programs has been described in $[18,23]$. These approaches permit re-execution of message-passing programs by periodically saving the states as well as the messages in transfer. Additionally, efficient techniques to avoid large amounts of checkpoint storage are included. The drawback of these approaches is that checkpointing is performed at predefined intervals. Thus, it may substantially affect the program's behavior, if the intervals are too narrow. In addition, a complex replay mechanism is needed to avoid inconsistent checkpoints, because program execution may only be initiated at consistent checkpoints.

In contrast, the method implemented in our approach does not suffer from the drawbacks above. By using the event graph model as a representation of parallel program behavior, checkpoints can be placed at virtually any place during the program's execution [21]. The user determines the size of the checkpoint interval by distributing checkpoints arbitrarily, and the system can easily verify checkpoint positions based on the happened-before relation. In addition, the event graph allows to describe arbitrary parallel programs, and is therefore not limited to the message-passing paradigm.

The method operates as follows: Again, the starting point is a given program execution described with an event graph model. The user selects suitable checkpoint positions by selecting arbitrary events of the graph. Upon selection of an event, the remainder of the checkpoint line can be automatically determined with the relations described by the event graph. Therefore, a set of local checkpoints is identified, which collectively serve as a place to obtain the state of the complete program. Besides that, the event graph contains all the required information to identify messages, which are crossing the checkpoint line and are therefore incomplete. In-transmit messages are sent before the checkpoint, but have not been received, while orphan messages are delivered before the sending process has reached the corresponding checkpoint.

An example for placing checkpoints is given in Figure 3. The graph under consideration is again the example finite element solver from Figure 1. As a simplification, we use the isolated group as displayed in Figure 2, although the same approach can be applied for the complete program execution as well. As shown in Figure 3, arbitrary 


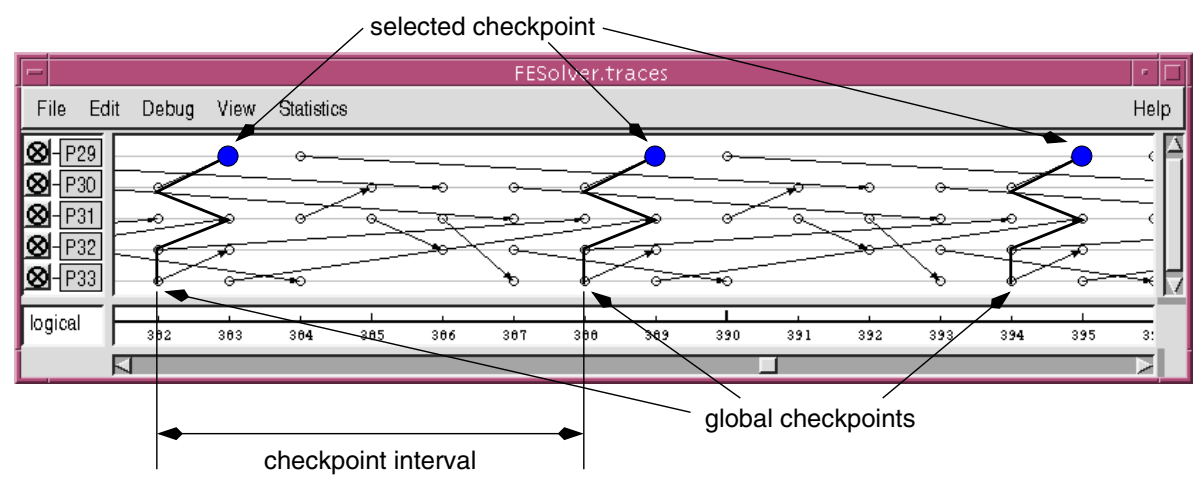

Fig. 3. Placement of checkpoints with the event graph model

local checkpoints have been selected on process P29. The remaining local checkpoints required to construct the global checkpoint are automatically determined by analyzing the event graph. In addition, orphan and in-transit messages are identified.

With the checkpoints defined as above, another execution of the program is initiated at the beginning. During this execution, the integrated checkpointing mechanism extracts the required checkpointing data and stores it to trace files:

$$
\text { event: } e_{p}^{i}=(p, i, \text { type }, \text { state })\left\{\begin{array}{l}
p \in \text { group } \\
e_{p}^{i}=\text { checkpoint }
\end{array}\right.
$$

Afterwards, the tracefiles contain the state of each process corresponding to the selected global checkpoint. During each subsequent execution cycle, the user may choose an arbitrary global checkpoint as a starting point for further investigations.

\section{Optional Extensions to the Debugging Strategy}

The technique described above with its two mechanisms is sufficient to achieve the goals of reducing the number of processes and the time axis during debugging. However, there are some problems existing for any parallel debugging tool, which must also be addressed by our debugging strategy. Two of these issues are the possibility of nondeterministic behavior and the perturbations due to monitor overhead. The event graph model offers some possibilities to decrease the effects of these problems.

The problem of possible nondeterministic behavior is that programs may deliver different results although the same input data is provided. As a consequence, subsequent iterations of the debugging cycle may be meaningless, because previously observed errors may occur only sporadically or vanish completely. This problem can be solved by using the data of the event graph as a prerequisite of the program's re-execution. If the behavior of the program is enforced corresponding to the events and relations contained in the event graph, an equivalent execution is obtained [11].

The perturbation due to the monitor overhead occurs due to the delay induced by the monitoring functionality. Consequently, a minimal monitor overhead reduces the probability of sustained perturbation effects. The most critical problems are changes in event 
order, which lead to different program behavior and thus different results. With the event graph model, monitor overhead does only occur when generating the event graph data. At this stage, only minimal information (as described in Section 2) is needed, which limits the monitor overhead. Any follow-up executions of the program are controlled by the data of the event graph, which prohibits any changes in event ordering [12]. Additional optimizations in this context are described in [13].

\section{Conclusions and Future Work}

Cyclic debugging of large-scale parallel programs with long-lasting execution times is hard and tedious, since repeated executions are needed to locate, backtrack, and eliminate erroneous behavior. The process isolation technique and an integrated checkpointing mechanism allows to alleviate this situation by reducing the number of processes and/or the execution time of subsequent debugging cycles. With this improvement, many programs which could not be adequately analyzed in the past due to their requirements of computational resources, are practicable.

The major drawback of the approach are certainly the memory requirements, which are defined by the needs to simulate the surroundings of the extracted process group or to initiate program execution at arbitrary intermediate points. While standard compression mechanisms offer initial support to decrease the amount of event graph data, more optimizations are certainly needed. These consumption of memory space as well as the applicability of the proposed techniques will be investigated in more detail for real-world projects. Fortunately, computational science offers a rich set of complex and large-scale applications, that need to be analyzed by parallel debugging tools.

Acknowledgments: This work was made possible by the support of our colleagues at the GUP Linz. We are most thankful to all of them, especially Christian Schaubschläger, who contributed a large amount to the implementation of the described debugging tools.

\section{References}

1. Agrawal, H., DeMillo, R.A., Spafford, E.H., An Execution Backtracking Approach to Debugging, IEEE Software, Vol. 8, No. 3, pp. 21-26 (May 1991).

2. Balzer, R.M., EXDAMS - EXtendable Debugging and Monitoring System, Proceedings of the AFIPS Spring Joint Computer Conference, pp. 567-580 (1969).

3. Bates, P., Wileden, J.S., High-Level Debugging of Distributed Systems: The Behavioral Abstraction Approach, Journal of Systems and Software, Vol. 3, No. 4, pp. 255-264 (Dec. 1983).

4. Choi, J.-D., Miller, B.P., Netzer, R.B., Techniques for Debugging Parallel Programs with Flowback Analysis, ACM Transactions on Programming Languages and Systems, Vol. 13, No. 4, pp. 491-530 (Oct. 1991).

5. Cunha, J.C., Loureno, J.M., Anto, T., An Experiment in Tool Integration: the DDBG Parallel and Distributed Debugger, EUROMICRO Journal of Systems Architecture, 2nd Special Issue on Tools and Environments for Parallel Processing, Elsevier Science Publisher (1998).

6. Etnus (Dolphin Interconnect Solutions Inc): TotalView 4.1.0, Documentation, Framingham, Massachusetts, USA, (2000).

http://www.etnus.com/pub/totalview/tv4.1.0/totalview-4.1.0-doc-pdf.tar 
7. Feldman, S.I., Brown, Ch.B., Igor: A System for Program Debugging via Reversible Execution, Proceedings of the ACM SIGPLAN and SIGOPS Workshop on Parallel and Distributed Debugging (May 1988), University of Wisconsin, Madison, Wisconsin, USA, SIGPLAN Notices, Vol. 24, No. 1, pp. 112-123 (January 1989).

8. Geist, G.A., Beguelin, A., Dongarra, J.J., Jiang, W., Manchek, R., and Sunderam, V.S., PVM3 - User's Guide and Reference Manual, Technical Report, Oak Ridge National Laboratory, Tennessee, MIT Press, Cambridge, MA, USA (1994).

9. Hood, R., The p2d2 Project: Building a Portable Distributed Debugger, Proc. SPDT'96, ACM SIGMETRICS Symp. on Par. and Distr. Tools, Philadelphia, USA, pp. 127-136 (May 1996).

10. Hossfeld, F., Teraflops Computing: A Challenge to Parallel Numerics, in: P. Zinterhof, M. Vajtersic, A. Uhl, (Eds.), "Parallel Computation", Proc. 4th Intl. ACPC Conf., Lecture Notes in Computer Science, Vol. 1557, Springer-Verlag, Salzburg, Austria, pp. 1-12 (Feb. 1999).

11. Kranzlmüller, D., Event Graph Analysis for Debugging Massively Parallel Programs, $\mathrm{PhD}$ Thesis, GUP Linz, Joh. Kepler Univ. Linz, Austria, (September 2000).

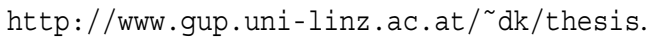

12. Kranzlmüller, D., Incremental Tracing and Process Isolation for Debugging Parallel Programs Computers and Artificial Intelligence, Vol. 19, No. 6, pp. 569-585 (Nov. 2000).

13. Kranzlmüller, D., Schaubschläger, Ch., Volkert, J., An Integrated Record\&Replay Mechanism for Nondeterministic Message Passing Programs, Proc. EuroPVM/MPI 2001, 8th European PVM/MPI Users' Group Meeting, Lecture Notes in Computer Science, Vol. 2131, Springer Verlag, Santorini, Greece, pp. 192-200 (September 2001).

14. Krawczyk, H., Wiszniewski, B., Analysis and Testing of Distributed Software Applications, in: Wilson, D.R., (Ed.), C3 - Industrial Control, Computers, and Communication Series, Research Studies Press Ltd., Baldock, Hertfordshire, England (1998).

15. Lamport, L., Time, Clocks, and the Ordering of Events in a Distributed System, Communications of the ACM, pp. 558 - 565 (July 1978).

16. May, J., Berman, F., Panorama: A Portable, Extensible Parallel Debugger, Proc. 3rd ACM/ONR Workshop on Parallel and Distributed Debugging, San Diego, CA, USA (May 1993), reprinted in: ACM SIGPLAN Notices, Vol. 28, No. 12, pp. 96-106 (Dec. 1993).

17. Message Passing Interface Forum, MPI: A Message-Passing Interface Standard - Version 1.1,(June 1995). http: / /www.mcs.anl.gov/mpi/

18. Netzer, R.H.B., Weaver, M.H., Optimal tracing and incremental reexecution for debugging long-running programs, Proc. ACM SIGPLAN Conference on Programming Language Design and Implementation, Orlando, FL, pp. 313-325(June 1994).

19. Plank, J.S., An Overview of Checkpointing in Uniprocessor and Distributed Systems, Focusing on Implementation and Performance, Technical Report of University of Tennessee, UT-CS-97-372, Jul. 1997.

20. van Rick, M., Tourancheau, B., The Design of the General Parallel Monitoring System, Programming Environments for Parallel Computing, IFIP, North Holland, pp. 127-137 (1992).

21. Thoai, N., Kranzlmüller, D., Volkert, J., Rollback-One-Step Checkpointing and Reduced MessageLogging for Debugging Message-Passing Programs, Proc. 5th International Meeting on Vector and Parallel Processing VECPAR2002, Porto, Portugal (June 2002). [submitted]

22. Weiser, M., Program Slicing, IEEE Transaction on Software Engineering, Vol. 10, No. 4, pp. 352-357 (July 1984).

23. Zambonelli, F., Netzer, R.H.B., An Efficient Logging Algorithm for Incremental Replay of Message-Passing Applications, Proc. 13th International Parallel Processing Symposium and 10th Symposium on Parallel and Distributed Processing (1999).

24. Zeller, A., Visual Debugging with DDD Dr. Dobb's Journal, No. 332, pp. 21-28 (2001). http://www.ddj.com/ articles/2001/0103/0103a/0103a.htm 